\title{
State density formalism of the Iwamoto-Harada model: A suitable tool to treat cluster emission from heavy-ion collisions with account for spin variables
}

\author{
Emil Běták ${ }^{1, a}$ and József Cseh² \\ 1 Institute of Physics SAS, 84511 Bratislava, Slovakia \\ ${ }^{2}$ MTA ATOMKI, P.O.B. 51, 4001 Debrecen, Hungary
}

\begin{abstract}
We study the possibility to include the cluster emission into the statistical pre-equilibrium (exciton) model suitable also for heavy ion collisions. The direct motivation of this paper is a possibility of producing superdeformed nuclei, which are easier to be detected in heavy-ion reactions than in those induced by light projectiles (nucleons, deuterons, $\alpha$-particles).
\end{abstract}

\section{Introduction}

The immediate request to calculate the particle (including cluster) emission in heavy-ion collisions (HIC) at modest energies come from nuclear structure studies concerning superdeformed and/or hyperdeformed nuclei of exotic shapes. The study of exotic nuclear shapes, in particular extreme deformations, is an interesting topisof the present-day research. Superdeformed (SD) states (with approximate ratio of the major axes of $2: 1: 1$ ) have been observed in several nuclei, while the appearance of hyperdeformation (HD), i.e., ellipsoidal shape of 3:1:1 is a hot topic for a discussion. Especially interesting is their existence in the $N=Z$ nuclei, in which the role of pairing, quartering, isospin, etc. can be studied.

As for the appearance of these shape isomers, usually different structure calculations give the same, or very similar results, thus providing us with a reliable prediction. Typically applied methods are those of the large-scale shell model, Nilsson-model, mean-field theories, cluster models, antisymmetrized molecular dynamics, etc.

Concerning the question of which reaction channels can populate these shape isomers, the answer is much less available. Not only reaction studies are missing, but also the connection between the structure and reaction channels is hardly known. In this respect a new symmetry-based method seems to be promising.

This method applies Nilsson-model in combination with quasi-dynamical SU(3) symmetry considerations. The shape isomers are obtained from a selfconsistency calculation concerning the quadrupole shape, or $\mathrm{SU}(3)$ symmetry $[1,2]$. The results are usually in very good agreement with the results of the traditional energyminimum calculations. The main advantage of this approach is that the $\mathrm{SU}(3)$ selection rule connects the shape isomers to binary clusterizations, i.e., to reaction channels [3]. In this way the likely reactions to populate the

\footnotetext{
a e-mail: betak@savba.sk
}

$\mathrm{SD}, \mathrm{HD}$, etc. states can be determined from the viewpoint of nuclear structure.

The reason why this surprisingly simple method works is twofold [4]: i) On the one hand the SU(3) symmetry, which is known to be a good approximate symmetry in the ground-state region, but it breaks down with increasing excitation, restore again for the SD, HD, etc., shapes (sometimes even better than for the ground states); $i i$ ) Furthermore, the relation of the shell and cluster structure, which is usually very simple in the ground state, but becomes complicated with increasing excitation energy, simplifies again for those shapes which have comensurate axes. There is already an experimental evidence for the existence of superdeformed nuclei of mass $A \leq 40$.

\section{Pre-equilibrium models and the generalized Iwamoto-Harada coalescence model}

\subsection{The exciton model and cluster coalescence}

We benefit from the fact that the pre-equilibrium exciton model (see, e.g. [5]), especially in its master-equation version, has a very high level of generalization irrespective of changing the reaction mechanism in the course of a nuclear reaction, as both the (equilibrated) compound nucleus and the fast direct reactions may be considered as limit cases of the model. Obviously, one has to pay for this consistency by losing many details, as it is the case in all statistical apoproaches.

As for the cluster emission, two diametrically different models were proposed within pre-equilibrium formalisms in early 70th, namely the idea of preformed $\alpha$-particles [6] and different versions of coalescence models; the first really applicable of them was the one by C.K. Cline [7]. The main difference between the two approaches stems from the fact that the $\alpha$-particle is a very compact entity, and it can be considered as one exciton (with specific mass, spin, etc.), whereas nucleons in coalescence models are all equal, and creating of a cluster by nucleon coalescence

(c) The Authors, published by EDP Sciences. This is an Open Access article distributed under the terms of the Creative Commons Attribution License 4.0 (http://creativecommons.org/licenses/by/4.0/). 
is a specific process which is essential for this group of approaches.

As the nucleons are much stronger coupled together within $\alpha$-particles than in any other near-by nucleus, the essential idea of the preformed $\alpha$-particles cannot be generalized to different types of clusters. The coalescence model, on the other hand, can be adapted to any light cluster from weakly-bound deuterons to strongly coupled $\alpha$ 's, and with some problems even to heavier clusters. We shall use the so-called Iwamoto-Harada (IH) coalescence model $[8,9]$ below, as it is probably the most justified one of the group of coalescence approaches.

\subsection{Iwamoto-Harada-(Bisplinghoff) coalescence model}

The basic idea of the IH model (and similar ones) is that not only excited nucleons can group together to form clusters, but both the excited ones as well as those from unexcited Fermi sea [8,9], i.e., the model includes a kind of statistical description of pickup.

In the following, we follow the notation of [8], which is fully consistent with the density-of-states concept inherent to the exciton model. Within the old coalescence model in the formulation of [10], the cluster emission rates of a cluster of type $x$ are proportional to

$$
\gamma_{x} \frac{\omega\left(p-p_{x}, h, U\right)}{\omega\left(p, h, E^{*}\right)} \frac{\omega\left(p_{x}, 0, \varepsilon_{x}+B_{x}\right)}{g_{x}},
$$

where $p_{x}$ and $g_{x}$ are the number of nucleons composing the cluster and the single-cluster density, respectively, $\gamma_{x}$ is the probability to form the cluster from given excitons and $\omega\left(p, h, E^{*}\right)$ is the density of states of $p$ particles and $h$ holes at excitation energy $E^{*}$. Further-on, $U$ is the excitation energy of the residual nucleus, $\varepsilon_{x}$ the energy of the emitted cluster and $B_{x}$ its binding (separation) energy in (from) the composite system ${ }^{1}$.

In the IH model, the density product $\omega(p-$ $\left.p_{x}, h, U\right) \omega\left(p_{x}, 0, \varepsilon_{x}+B_{x}\right)$ is replaced by the folding expression

$$
\begin{array}{r}
\sum_{p^{*}=1}^{p_{x}} \int_{\varepsilon_{x}+B_{x}}^{E^{*}} \omega\left(p-p^{*}, h, E^{*}-\varepsilon_{1}\right) \omega\left(p^{*}, 0, \varepsilon_{1}\right) \times \\
\omega\left(0, p_{x}-p^{*}, \varepsilon_{2}\right) \mathrm{d} \varepsilon_{1}
\end{array}
$$

where $p^{*}$ is the number of particle excitons contributing to the formation of the cluster, and the remaining ( $p_{x}-$ $\left.p^{*}\right)$ nucleons are picked up from the Fermi sea. The cluster density $g_{x}$ is proportional to the cluster formation probability $\gamma_{x}$ [8], what makes the formulation of the problem - as far as complex particles (clusters) concerns — to be parameterless.

Further improvements or generalizations of the model included the Bisplinghoff's suggestion [11] that not all nucleons are available for the cluster formation, but only those close to the Fermi energy, and the energy width of the "band of availability" is determined by the binding energy of nucleons within the cluster [11]. As the binding energy of nucleons in a deuteron is small, the pickup

\footnotetext{
${ }^{1}$ The sign of the binding or separation energy is not unique in all publications. Here, we use the convention $B_{x}>0$ for the bound and $B_{x}<0$ for the unbound particle.
}

possibility of nucleon coalescence to form a deuteron is of minor effect. Thus, strongly bound entities like $\alpha$ 's, have large energy space available for pickup (which makes the approach close to the original ideas $[8,9]$ ), whereas the loosely coupled objects (e.g. deuterons) get rather close to the standard coalescence model (see [12]).

Updates of the model include the modification to keep the consistency between the compound nucleus (Weisskopf-Ewing) formulae and the exciton model [13] not only for nucleons, but also for the cluster emission [12, 14], statistical "equivalent" of knockout [14] and some others $[14,15]$.

\section{Heavy-ion induced reactions and the exciton model}

The main problem of being able to calculate the preequilibrium emission from heavy ion reactions using the exciton model is to find a feasible way to get the initial exciton configuration. Essentially, this can be done by calculating the overlap of the colliding nuclei in their momenta space $[16,17]$. For practical use, however, a simple empirical formula is preferred instead of trajectory and overlap calculations.

For not too high energies, the suitable one for the initial exciton number $n_{0}$ is

$$
\frac{n_{0}}{A_{P}}=0.09+\left(0.38-0.08 \cdot \frac{A_{T}-A_{P}}{A_{T}+A_{P}}\right) \cdot \sqrt{\frac{E_{c m}-V_{C}}{A_{P}}},
$$

where $V_{C}$ is the Coulomb barrier and the subscripts $T$ and $P$ denote the target and projectile, respectively $[18]^{2}$.

At higher energies, say above $10 \mathrm{MeV} /$ nucleon above the Coulomb barrier, another form is more appropriate, namely

$$
E^{*} / n_{0}=4.6+0.54\left(E_{c m}-V_{C}\right) / A_{P}
$$

as the parametrization of model calculations [17], or

$$
E^{*} / n_{0}=6.8+0.54\left(E_{c m}-V_{C}\right) / A_{P}
$$

as the fit to the data [19]. As the $n_{0}$ values vs energy according to Eq. (4) cross those from Eq. (5), we preferred to use the intersection energy of both curves as the switching point from one formula to the other one, instead of somewhat arbitray value of $10 \mathrm{MeV} /$ nucleon.

One important step was introduced later on, namely the phenomenological way to account for the projectile breakup [20]. The formulae presented therein are surprisingly stable and practically independent of the binding energy of nucleons within the cluster, reaction energy etc.. Therefore we dared to introduce projectile breakup in our calculations using this suggestion, even though the breakup part should be taken with high care.

\subsection{Heavy-ion reactions and the exciton model with angular momentum}

The inclusion of angular momentum is essential mainly for proper treatment of $\gamma$ de-excitation and in the heavyion collisions. It was introduced into the pre-equilibrium

2 The formula assumes that $A_{P} \leq A_{T}$. 
calculations by Obložinský and Chadwick [21] and applied in the PEGAS computer code [22]. However, only the nucleon- and/or $\alpha$-particle-induced reactions were considered at that time.

The idea on how to describe HIC appeared in [23], namely that one has to subtract the double nuclear system rotational energy from the available energy in the calculation of the inital exciton configuration $n_{0}$,

$$
E_{\mathrm{cm}}^{\prime}(l)=E_{\mathrm{cm}}-E_{\mathrm{rot}}(l),
$$

and it was soon implemented in the updated versions of computer codes (see, e.g., [24]). The rotational energy $E_{\mathrm{rot}}(l)$ should be expressed using the inertia momentum of two touching spheres, as the time-dependent calculations [25] indicated that - at least at modest energies, say $5-20 \mathrm{MeV} / \mathrm{A}$ - the distance of the centers of the colliding nuclei remains practically unchanged after the sticking phase of the reaction for a while, which is much longer than the time typical for the pre-equilibrium emission $[23,25]$.

However, all this was only for the nucleon and $\gamma$ emission only. Here we report on the first steps to consider pre-equilibrium (exciton model) cluster emission in heavyion collisions.

\section{Super- and hyperdeformed nuclei and the choice of reaction}

We take ${ }^{36} \mathrm{Ar}$ production for the initial calculations. Therein, the calculations predict its existence also in super- and hyperdeformed state [1]. We sketch here, how the superdeformed and the hyperdeformed states of the nucleus can be populated in a single-alphaemitting reaction, namely ${ }^{20} \mathrm{Ne}+{ }^{20} \mathrm{Ne} \rightarrow{ }^{36} \mathrm{Ar}(\mathrm{SD})+\alpha$ and ${ }^{20} \mathrm{Ne}+{ }^{20} \mathrm{Ne} \rightarrow{ }^{36} \mathrm{Ar}(\mathrm{HD})+\alpha$.

The U(3) selection rule has to be applied, and the relevant quantum numbers are [1]: ${ }^{20} \mathrm{Ne}:[12,4,4]$, ${ }^{36} \mathrm{Ar}(\mathrm{SD}):[32,16,8],{ }^{36} \mathrm{Ar}(\mathrm{HD}):[48,8,8],{ }^{4} \mathrm{He}:[0,0,0]$. The following sets of equations express the matching of the structural and reaction symmetries [26]:

$$
\begin{array}{r}
{[12,4,4] \otimes[12,4,4] \otimes[16+i, 0,0]=} \\
{[32,16,8] \otimes[0,0,0] \otimes[i, 0,0] \oplus \ldots}
\end{array}
$$

for the SD state, and

$$
\begin{array}{r}
{[12,4,4] \otimes[12,4,4] \otimes[24+j, 0,0]=} \\
{[48,8,8] \otimes[0,0,0] \otimes[j, 0,0] \oplus \ldots}
\end{array}
$$

for the hyperdeformed state, where the minimum of the relative motion quantum numbers of $[n, 0,0]$ is determined by the Pauli-principle: $i, j=8,9,10, \ldots$.

\section{Results}

As suggested above, we have taken ${ }^{20} \mathrm{Ne}+{ }^{20} \mathrm{Ne}$ collision leading to the ${ }^{40} \mathrm{Ca}$ composite system (composite system $=$ system generally not in equilibrium), which after the emission of an $\alpha$ particle (or two deuterons, or a deuteron plus neutron plus proton, or two neutrons and two protons in arbitrary order) transforms into ${ }^{36} \mathrm{Ar}$, which is assumed to exist also in a SD and in HD shapes. For the very beginning, we performed simple (and fast) calculations
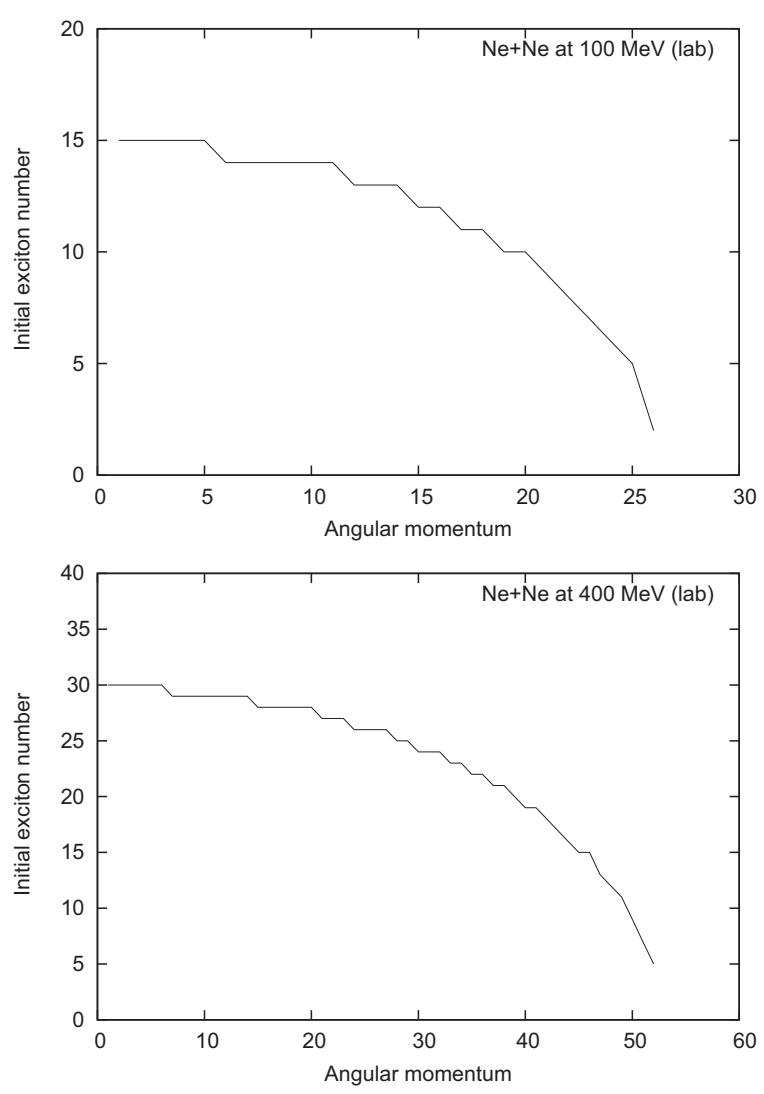

Figure 1. Calculated initial exciton number for different angular momentum for the ${ }^{20} \mathrm{Ne}+{ }^{20} \mathrm{Ne}$ reaction at $E_{l a b}=100 \mathrm{MeV}$ (top) and $E_{l a b}=400 \mathrm{MeV}$ (bottom). Note that the exciton number is an integer.

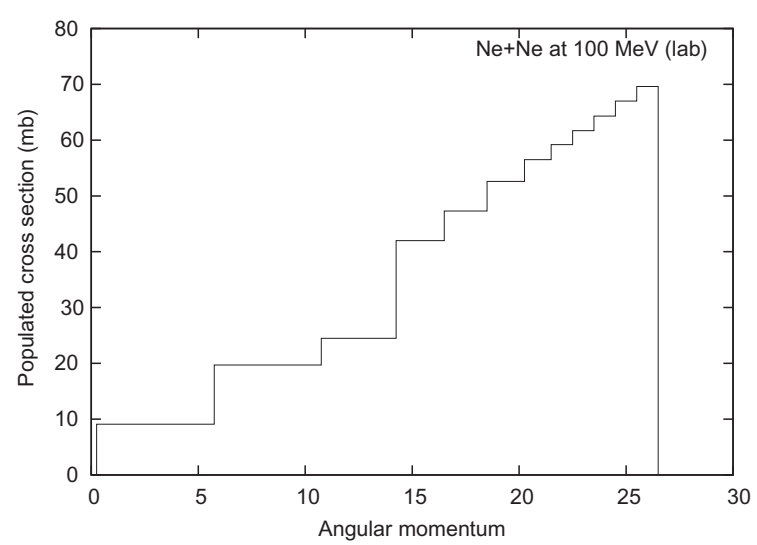

Figure 2. Partial contributions of different partial waves to the composite system (i.e., ${ }^{40} \mathrm{Ca}$ ) formation cross section for the the same reaction as in the preceding figure (at $100 \mathrm{MeV}$ ). (Please, put attention that the contributions to the cross sections are not given by the height, but by the area below the histogram!)

without considering the angular momentum variables, just to see, how the yields vary with chosing different emission sequence and how the competition of channels depends on energy. This is to enable to estimate the best region for the ${ }^{36}$ Ar population.

Figure 1 brings the energy dependence of the ${ }^{36} \mathrm{Ar}$ production cross section from the ${ }^{20} \mathrm{Ne}+{ }^{20} \mathrm{Ne}$ reaction, together with contributions of different reaction chains, at 
this time not yet considering angular momentum variables, but calculated using more simple code PEQAG [27] (updated and enlarged for the possibility of heavy-ion collisions).

\section{Conclusions}

The calculations of the ${ }^{36} \mathrm{Ar}$ production cross sections (in fact, the cross sections for the emission of nucleons, $\gamma$ 's and clusters) within the spin-dependent version of the exciton model code are in progress. As the second step, we envisage to differentiate between the ground state and the SD one, and to calculate their production separately. An easy way to determine the portion of superdeformed nuclei from the total argon production is the use of master equation in the same way as was suggested by Moretto and Schmitt [28] for isotope production (see also [29]).

The authors are grateful to the referee for his/her comments, which significantly helped to improve the form and understanding of the paper. The work work has been supported in part by the VEGA and APVV grants Nos. 2/0110/14 and 15-0225, respectively, and by the Hungarian Research Fund OTKA Grant No. K112962, and also by the billateral exchange program of the Slovak and the Hungarian Academies of Sciences.

\section{References}

[1] J. Cseh, J. Darai, W. Sciani, Y, Otani, A. Lepine-Szily, E.A. Benjamin, L.C. Chamon and R.L. Filho, Phys. Rev. C80, 034320 (2009)

[2] J. Darai, J. Cseh, N.V. Antonenko, G. Royer, A. Algora, P.O. Hess, P.V. Jolos qand W. Scheid, Phys. Rev. C84, 024302 (2011); J. Darai, J. Cseh and D.G. Jenkins, ibid. C86, 064309 (2012)

[3] J. Cseh, A. Algora, J. Darai and P.O. Hess, Phys. Rev. C70, 034311 (2004); A. Algora, J. Cseh, J. Darai and P.O. Hess, Phys. Lett. B639, 451 (2006)

[4] J. Darai, J. Cseh, G. Adamian and N. Antonenko, EPJ Web of Conf. 38, 16001 (2012); J. Darai, J. Cseh, J. Phys. Conf. Ser. 580, 012057 (2015)

[5] E. Gadioli and P.E. Hodgson, Pre-Equilibrium Nuclear Reactions, (Clarendon Press, Oxford, 1992)

[6] L. Milazzo-Colli and G.M. Braga-Marcazzan, Phys. Lett. B36, 447 (1971); L. Colli-Milazzo and G.M. Marcazzan-Braga, ibid. B38, 155 (1972)

[7] C.K. Cline, Nucl. Phys. A193, 417 (1972)
[8] J. Dobeš and E. Běták, in Int. Conf. Reaction Models 77, Balatonfüred 1977, Ed. L.P. Csernai, p. 195 (Budapest, 1977)

[9] A. Iwamoto and K. Harada, Phys. Rev. C26, 1821 (1982)

[10] I. Ribanský and P. Obložinský, Phys. Letts. B45, 318 (1973)

[11] J. Bisplinghoff, Phys. Rev. C50, 1611 (1994)

[12] E. Běták, Int. J. Mod. Phys. E13, 63 (2004)

[13] I. Ribanský, P. Obložinský and E. Běták, Nucl. Phys. A205, 545 (1973)

[14] E. Běták, AIP Conf. Proc. 769, 1168 (2005)

[15] C. Kalbach, Phys. Rev. C71, 034606 (2005); C. Kalbach Walker, AIP Conf. Proc. 769, 1180 (2005)

[16] E. Fabrici, E. Gadioli, E. Gadioli Erba, M. Galmarini, F. Fabbri and G. Reffo, Phys. Rev. C40, 2548 (1989); E. Fabrici, E. Gadioli, E. Gadioli Erba and M. Galmarini, ibid. C42, 2163 (1990)

[17] N. Cindro, M. Korolija, E. Běták and J.J. Griffin, Phys. Rev. Lett. 66, 868 (1991)

[18] N. Cindro, E. Běták, M. Korolija and J.J. Griffin, Fizika B1, 51 (1992)

[19] M. Korolija et al., Phys. Rev. Letts. 60, 193 (1988)

[20] C. Kalbach Walker, in FENDL-3 Library. Final Report of the CRP on Nuclear Data Libraries for Advanced Systems: Fusion Devices, Report INDC(NDS)-0645, p. 11 (IAEA, Vienna, 2013)

[21] P. Obložinský, Phys. Rev. C35, 417 (1987); P. Obložinský and M.B. Chadwick, ibid. 42, 1652 (1990)

[22] E. Běták and P. Obložinský, PEGAS: PreEquilibrium-Equilibrium Gamma-And-Spin Code (PC Version). Report INDC(SLK)-001 (IAEA, Vienna, 1993)

[23] E. Běták, Fizika B12, 11 (2003)

[24] E. Běták, AIP Conf. Proc. 1491, 342 (2012)

[25] E. Běták and V.D. Toneev, Yad. Fiz. 38, 1179 (1983); English version Sov. J. Nucl. Phys. 38, 710 (1983)

[26] J. Darai, J. Cseh, A. Algora, Int. J. Mod. Phys. E20, 815 (2011)

[27] E. Běták, PEQAG: A PC version of fully preequilibrium computer code with gamma emission. Report INDC(CSR)-016/LJ (IAEA, Vienna, 1989)

[28] L.G. Moretto and R. Schmitt, J. Phys. Coll. C5, 109 (1976); ibid., p. 140

[29] E. Běták and V.D. Toneev, J. Phys. G9, L47 (1983) 